\title{
Model of End Stage Liver Disease Score and Hepatocellular Carcinoma
}

\author{
HANY SHABANA, M.D. ${ }^{1}$; MOHAMED ASKAR, M.D. ${ }^{2}$; MOHAMED ELRAKHAWY, M.D. ${ }^{\mathbf{3}}$ and \\ MOHAMED HARAS, M.D. 4 \\ The Department of Internal Medicine, Hepatology \& Gastroenterology Unit, Specialized Medical Hospital, \\ Faculty of Medicine, Mansoura University ${ }^{1}$, Blood Bank, Aga Hospital, Ministry of Health ${ }^{2}$, Radiology Department, \\ Specialized Medical Hospital, Faculty of Medicine, Mansoura University ${ }^{3}$ and Clinical Pathology Department, \\ Faculty of Medicine, Mansoura University4, Mansoura, Egypt
}

\begin{abstract}
Background: Hepatocellular carcinoma (HCC0 is the most common primary hepatic malignancy worldwide [1]. It is the first cause of cancer mortality in Egypt [2] due tothe heavy burden of chronic HCV (14.7\%). Among cirrhotic patients, 1-4\% per year will develop HCC [3]. HCC patients evaluated for liver transplantation are often given exceptional MELD score, giving them a priority for liver transplantation. We aimed at determining the MELD score in HCC patients, its correlation with TNM tumour stage and tumour size. Also, we aimed at determining a cut off value of MELD score above which chronic HCV (CHC) cirrhotic patients have high chance to develop HCC.
\end{abstract}

Aim of Work: To determine the actual MELD score in HCC patients, its correlation with TNM stage and tumour size. Also, we aimed at termining a cut off value of MELD score above which chronic HCV (CHC) cirrhotic patients have a high chance to develop HCC.

Material and Methods: The study included 98 patients with $\mathrm{CHC}$ and $\mathrm{HCC}$ (group I) and 219 patients with CHC without HCC (group II). CHC was diagnosed by ELISA for HCV Antibody and serum HCV RNA. HCC diagnosis was based on EASL criteria i.e. focal hepatic lesion with arterial phase enhancement and washout in portal and delayed phases, obtained by contrast enhanced abdominal CT and/or MRI. HCC was staged according to the seventh edition TNM tumour staging system. MELD score was calculated using the following formula: MELD score $=10 *[(0.957 *$ In (Creatinine $)]+$ $[0.378 *$ In (Bilirubin) $]+[1.12 * \operatorname{In}(\mathrm{INR})]+6.43$. We used the MELD score calculator of the liver application of the EASL. We computed ROC curve for MELD score concerning the prediction of HCC. Stratum specific likelihood ratio (SSLR) was calculated as the pro-portion of diseased subjects (HCC) with a test result in a given range divided by the proportion of non-diseased subjects (non HCC) with a test result in the same range [4].

Results: MELD score was significantly higher in group I than group II. The score was $9.71 \pm 4.08$ in group I versus $5.61 \pm 3.25$ in group II $(p \leq .000)$. In group I the MELD score

Correspondence to: Dr. Hany Shabana, The Department of Internal Medicine, Specialized Medical Hospital, Faculty of Medicine, Mansoura University, Mansoura, Egypt ranged from 0.7 to 20.33 . There was significant positive correlation between MELD score and TNM tumour stage ( $r=0.312, p=0.002$ ) but the correlation was insignificant as regards the tumour size ( $r=0.041, p=0.687$ ). The distribution of TNM tumour stage in group I was as follows: Stage I represented $19.3 \%$, stage II represented $25.5 \%$, stage IIIa represented $19.3 \%$, stage IIIb represented $18.3 \%$, stage IIIc represented $1 \%$, stage IVa represented $8 \%$ and stage IVb represented $7 \%$. The cut off value of MELD score above which there was a high risk of HCC development was $\geq 5.74$ The area under the curve (AUC) was $78.3 \%$, sensitivity was $87.8 \%$, specificity was $56 \%$, positive predictive value (PPV) of $46.7 \%$, negative predictive value (NPV) of $91 \%$, accuracy of $65.3 \%$ and positive likelihood ratio (LR) of 1.96 . The SSLR for HCC presence by MELD score was 0.21 in score <5, 0.97 in score from 5 to 10 and 4.57 in score $>10$.

Conclusion: MELD score has significant positive correlation with TNM tumour stage in HCC cases. CHC patients with MELD score >10 have SSLR for HCC presence of 4.57 and are in need for closer follow-up.

Key Words: End stage - Liver disease - HepatocellularCarcinoma.

\section{Introduction}

HEPATOCELLULAR carcinoma (HCC) is the most common primary hepatic malignancy worldwide [1]. It is the first cause of cancer mortality in Egypt [2] due to the heavy burden of chronic HCV (14.7\%). Among cirrhotic patients, $1-4 \%$ per year will develop HCC [3]. Mazzaferro and colleagues reported that liver transplantation for the treatment of small HCC, defined as 1 lesion smaller than $5 \mathrm{~cm}$ or 3 lesions smaller than $3 \mathrm{~cm}$ (Milan criteria), produced a 4-year recurrence-free survival rate of $83 \%$ [4] . The results of this study have led to the acceptance of liver transplantation as a main treatment modality for surgically unresectable HCC. The MELD score, initially developed to determine prognosis following a trans-jugular intra-hepatic shunt (TIPS) procedure for liver failure [ $\boldsymbol{\Re}$, is now widely used in the liver transplant centers to pri- 
oritize deceased donor liver allocation. It is a logarithmic score that is comprised of International Normalized Ratio (INR), serum creatinine, total serum bilirubin and the etiology of cirrhosis. A modification of the MELD score formula with the variable for etiology of cirrhosis excluded [6], was adopted by the United Network of Organ Sharing (UNOS) in February 2002 as the standard by which transplant recipients are prioritized because higher score is associated with shorter survival. The MELD based allocation system, implemented in 2002, assigns "exception" scores for patients with HCC within the Milan criteria. Patients with HCC were granted a MELD exception score of 22 at listing, with additional points granted every 3 months [7] The HCC exception score was intended to reflect candidates' expected waitlist mortality due to progression of the tumor. It was quickly determined that the initial scores assigned to waitlisted HCC candidates overestimated the likelihood of disease progression and/or death while waiting, and the policy was adjusted to decrease the score in 2003 and again in $2005[\mathbf{8 , 9}]$. The current system of allocation for candidates with HCC has been in place since the 2005 adjustment. Even under this policy, analyses of waitlist survival demonstrate that candidates with HCC are much less likely than candidates without HCC to die or to be removed from the list while waiting [10]. In addition, candidates with HCC undergo transplant at a higher rate than candidates without HCC, indicating a substantial advantage over non-HCC candidates, who principally have complications of end-stage liver disease and thus high native MELD scores [11] Despite the increased transplant rate, post-transplant survival for patients with HCC remains inferior to survival of patients without HCC [12,13]. So, various proposals are taken to make the allocation system more equitable between candidates with and without HCC. The expert panel behind the new guidelines reports that there was general consensus among the members for a need to develop a "calculated continuous HCC priority score for ranking $\mathrm{HCC}$ candidates" who are awaiting liver transplants. A calculated continuous HCC priority score incorporates calculated MELD, alphafetoprotein (AFP), tumor size, and rate of tumor growth. Only candidates with at least stage T2 tumors will receive additional HCC priority-score points. The candidate must meet the Milan criteria for a minimum of 3 months before additional priority-score points are assigned [14]

\section{Aim of work:}

To determine the actual MELD score in HCC patients, its correlation with TNM stage and tumour size. Also, we aimed at determining a cut off value of MELD score above which chronic HCV(CHC) cirrhotic patients have a high chance to develop HCC.

\section{Patients and Methods}

The study was conducted in the Specialized Medical Hospital, Mansoura University in the period from November 2014 to June 2015 and included 98 patients with CHC and HCC (group I) and 219 patients with $\mathrm{CHC}$ without $\mathrm{HCC}$ (group II). CHC was diagnosed by fourth generation ELISA for HCV Antibody and confirmed by measurement of serum HCV RNA quantity. HCC diagnosis was based on European Association of The Study Of Liver Disease (EASL) criteria i.e. focal hepatic lesion with enhancement in arterial phase and washout in portal and delayed phases, obtained by contrast enhanced abdominal CT and or MRI [15] HCC was staged according to the seventh edition TNM HCC staging system as follows [16]

\section{Primary tumor $(T)$ :}

- TX: Primary tumor cannot be assessed.

- T0: No evidence of primary tumor.

- T1: Solitary tumor without vascular invasion.

- T2: Solitary tumor with vascular invasion or multiple tumors, none more than $5 \mathrm{~cm}$.

- T3a: Multiple tumors more than $5 \mathrm{~cm}$.

- T3b: Single tumor or multiple tumors of any size involving a major branch of the portal vein or hepatic vein.

- T4: Tumor(s) with direct invasion of adjacent organs other than the gallbladder or with perforation of visceral peritoneum

Notes:

- Vascular invasion includes gross or microscopic involvement.

- For T3, a major branch means the right or left portal vein, or right-middle or left hepatic vein.

- Multiple tumors includes satellites, multifocal tumors and intrahepatic metastases.

Regional lymph nodes $(N)$ :

- NX: Regional lymph nodes cannot be assessed.

- N0: No regional lymph node metastasis.

- N1: Regional lymph node metastasis.

\section{Distant metastasis (M):}

- M0: No distant metastasis.

- M1: Distant metastasis. 
Stage grouping:

- I: T1 N0 M0.

- II: T2 N0 M0.

- IIIA: T3a N0 M0.

- IIIB: T3b N0 M0.

- IIIC: T4 NO M0.

- IVA: Any T N1 M0.

- IVB: Any T any N M1.

\section{formula:}

MELD score was calculated using the following

$9.6 \times($ creatinine $\mathrm{mg} / \mathrm{dl})+3.8 \times$ (bilirubin $\mathrm{mg} / \mathrm{dl})$ $+11.20 \times(\mathrm{INR})+$ 6.4.(17). The MELD score calculator of the iLiver application of the EASL was used for MELD score calculation. ROC curve for MELD score concerning the prediction of HCC was computed. Stratum specific likelihood ratios
(SSLR) of MELD score in relation to HCC was calculated as the proportion of diseased subjects with a test result in a given range divided by the proportion of non-diseased subjects with a test result in the same range. The percentiles method for calculation of the SSLR was used as follows:

Step 1: Establish the strata and tabulate the stratum specific test results.

Step 2: Compute proportion of patients with the disease with that results.

Step 3: Compute proportion of patients without the disease with that results.

Step 4: Divide the fractions with the disease by the fractions without the disease. SSLR greater than 1 indicate the test result occurs more frequently in those in whom disease is present than it does in those in whom it is absent [18].

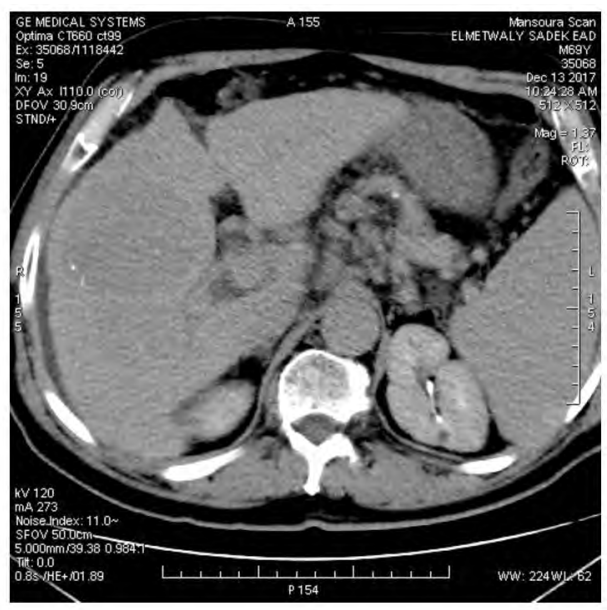

(B)

Fig. (1): Triphasic CT scan of the liver shows multifocal hcc of the right liver lobe that show heterogeneous enhancement on arterial phase and rapid washout on delayed phase.

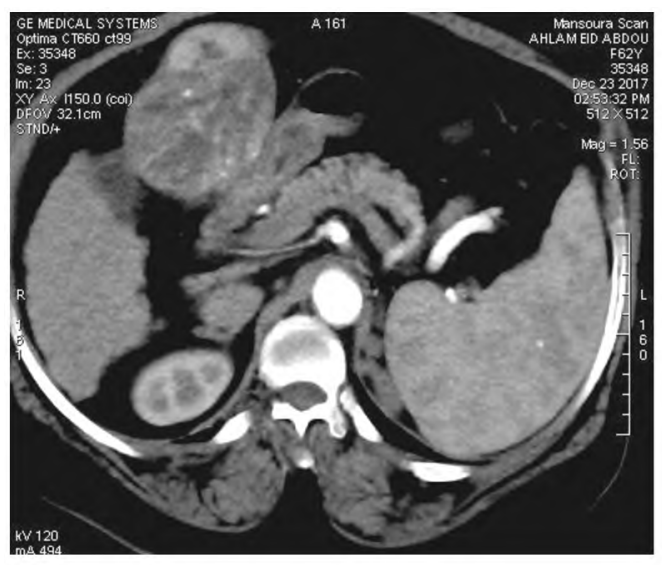

(A)

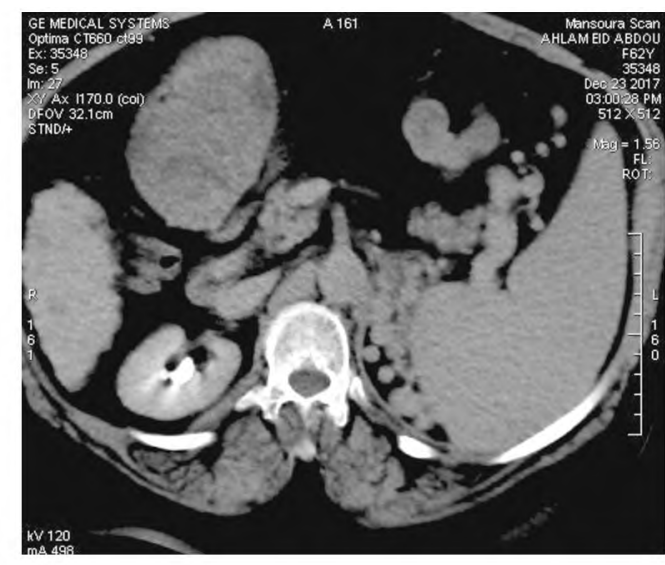

(B)

Fig. (2): Triphasic CT scan of the liver shows large exophytic left lobe hcc that show heterogeneous enhancement on arterial phase and rapid washout on delayed phase. 


\section{Results}

There was strong male predominance in HCC group where males represented $90.1 \%$ of cases while females represented $9.9 \%$ of cases. The age of the patients was significantly higher in HCC group than non HCC group ( $p$-value $=.000)$. The age of the patients at $\mathrm{HCC}$ presentation was $59.34 \pm 7.61$ years. The presence of Type $2 \mathrm{DM}$ was significantly higher in HCC cases (23.6\%) than non HCC cases $(11.4 \%)(p=.01)$. HCC was mono-focal in $33.3 \%$, multifocal in $63.1 \%$ and diffuse infiltrative in $3.6 \%$ of cases. The AST, serum bilirubin, INR, serum creatinine and CTP score were significantly higher in group 1 than group 2. $p$-values were .007, $.000, .000, .000, .000$ respectively. The serum Albu$\mathrm{min}$ and platelet count were significantly lower in group1 than group 2 ( $p$-value was .000 in both).

The distribution of TNM HCC stage in group I was as follows: Stage I represented 19.3\%, stage II $25.5 \%$, stage IIIa $19.3 \%$, stage IIIb $18.3 \mathrm{C}$, stage IIIc $1 \%$, stage IVa $8 \%$ and stage IVb 7\%. MELD score was significantly higher in group I than group II. The actual (laboratory) MELD score was $9.71 \pm 4.08$ in group I versus $5.61 \pm 3.25$ in group II ( $p$-value $\leq .000)$. In group I the MELD score ranged from 0.7 to 20.33 points. It was more than 10 in $48 \%,>5 \leq 10$ in $41.8 \%$ and $\leq 5$ in $10.2 \%$ in group I. There was significant positive correlation between MELD score and TNM HCC stage ( $r=0.312$, $p=0.002$ ) but the correlation was insignificant as regards the tumour size ( $r=0.041, p=0.687)$.

The cut off value of MELD score above which there was a high risk of HCC development was $\geq 5.74$. The area under the curve (AUC) was $78.3 \%$, sensitivity was $87.8 \%$, specificity was $56 \%$, positive predictive value (PPV) of $46.7 \%$, negative predictive value (NPV) of $91 \%$, accuracy of $65.3 \%$ and positive likelihood ratio (LR) of 1.96.

The SSLR for HCC presence by MELD score was 0.21 if the MELD score was $<5,0.97$ points, 0.97 if the MELD score was from 5 to 10 and 4.57 if the MELD score was $>10$.

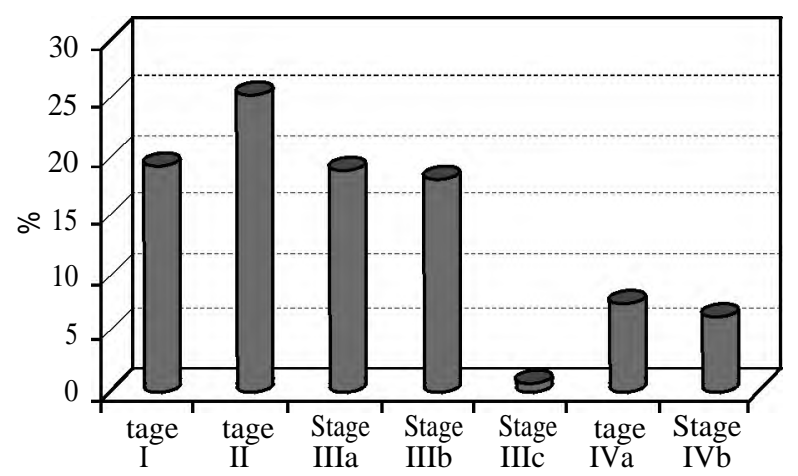

Fig. (3): Distribution of TNM HCC stage in group 1 patients.

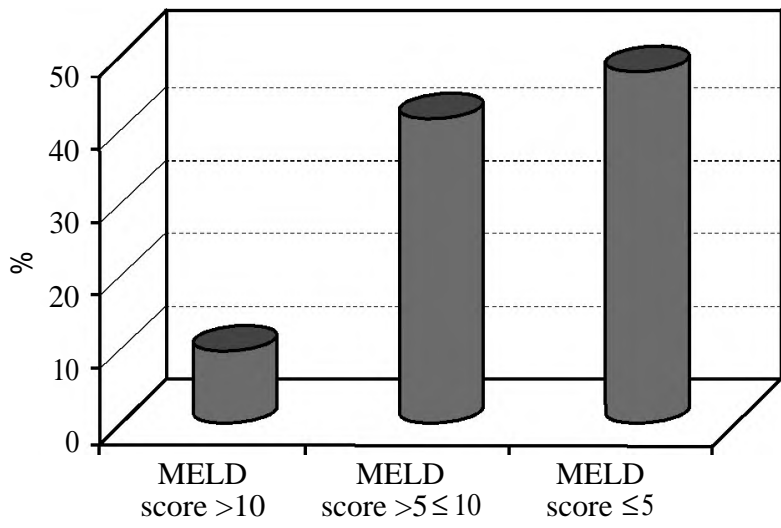

Fig. (4): Distribution of MELD score in group 1 patients.

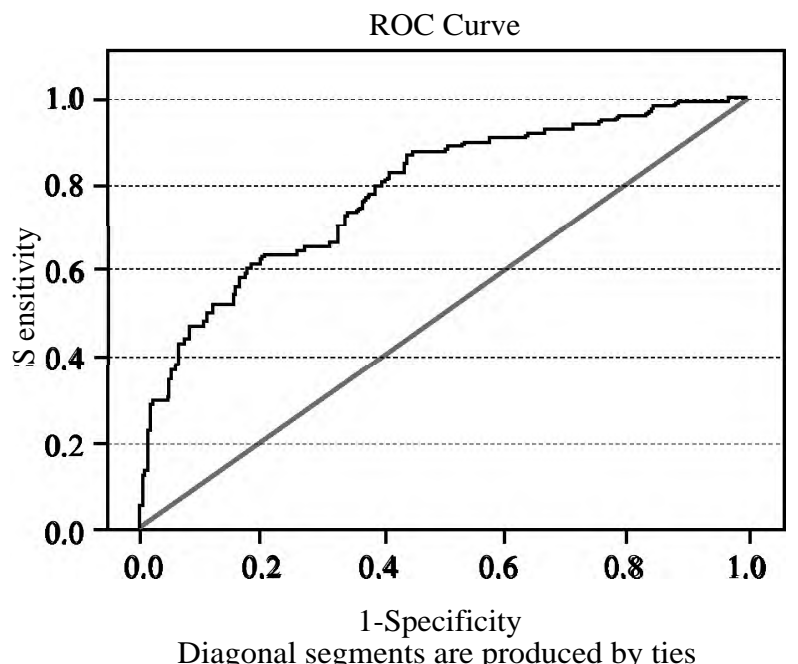

Fig. (5): ROC analysis curve of MELD score and HCC.

\section{Discussion}

HCC patients within Milan criteria are always given exceptional MELD score points giving them a priority for liver transplantation over non $\mathrm{HCC}$ cases. However, their outcome and post-transplantation survival are not superior to non HCC cases. Also, Patients with HCC had a lower dropout rate from the waitlist compared with patients without HCC. So, various proposals are taken to make the allocation systems more equitable between patients with and without HCC. Recently, calculated continuous priority HCC score involving the calculated MELD score, AFP level, tumour size and rate of tumour growth are developed.

In the present work, the age of $\mathrm{CHC}$ induced $\mathrm{HCC}$ patients was significantly higher than $\mathrm{CHC}$ patients without HCC. This can be explained by the fact that $\mathrm{HCC}$ mostly occurs in CHC patients with advanced fibrosis or cirrhosis, which takes a long time to occur in $\mathrm{CHC}$ patients due to the slowly progressive course of the disease. There was a strong male predominance in HCC cases 
which may be attributed to hormonal factors and more exposure of males to $\mathrm{CHC}$ infection. The presence of type $2 \mathrm{DM}$ was significantly higher in HCC than non HCC cases It is now well established that the risk of cancer is increased in patients with type 2 diabetes, including HCC [19]. Also, preexisting diabetes was significantly associated with an increased incidence of HCC and HCC specific mortality [20]. HCC was diagnosed while the tumour was mono-focal in $33 \%$ of cases and at early stages (TNM stage I and II) in $44.8 \%$ of cases. This can be attributed to the application of surveillance programme using abdominal Ultrasonography every 6 months to high risk populations (CHC patients with advanced fibrosis or cirrhosis). The CTP and MELD scores were significantly worse in HCC than non $\mathrm{HCC}$ cases which may be explained by the more advanced liver fibrosis stage at the time of HCC diagnosis and the deleterious effect of the tumour itself on the liver parynchema and hepatic functions. The actual (laboratory) MELD score in HCC cases ranged from 0.7 to 22 points with mean score of 9.71 points \& standard deviation of 4.08 points. It was less than 10 points in $52 \%$ of cases. Also, HCC was diagnosed at TNM stage I in 19.3\% of cases. These findings and the presence of significant positive correlation between the laboratory MELD score and TNM HCC stage support the recent trend of giving exceptional MELD score points to HCC patients with tumours of at least T2 stage only [14]. These findings also support the new policy, which differ from that implemented in October 2015 [21]. In the new policy, HCC patients are listed with their native MELD score for the first 6 months and If patients are still within criteria after 6 months, they are granted a MELD score of 28 , which will increase every 3 months to a maximum of 34 points [21]

These findings may be helpful in countries where cadaveric liver transplantation and organ allocation systems are applied. In Egypt, the living donor liver transplantation is the main method used for liver transplantation and cadaveric liver transplantation is still not applied due to regulatory causes.

Using ROC curve, the cut off value of MELD score above which there was a high risk of HCC development was $\geq 5.74$ with positive likelihood ratio (LR) of 1.96. In clinical practice, however, MELD score will not be used as a diagnostic test of HCC, but used as an indicator of the risk of HCC. In this aspect, SSLR is better than a fixed cutoff value [22]. The SSLR for HCC presence by MELD score was 4.57 if the MELD score was $>10$. This means that MELD score $>10$ is 4,57 times more likely to occur in $\mathrm{CHC}$ patients with $\mathrm{HCC}$ than CHC patients without HCC. So, CHC patients expressing MELD score $>10$ are in need for much closer follow-up for earlier detection of $\mathrm{HC} \mathrm{C.}$

\section{Conclusion:}

MELD score has significant positive correlation with TNM HCC stage. MELD score was less than 10 points in $52 \%$ of chronic $\mathrm{HCV}$ induced $\mathrm{HCC}$ cases. Chronic hepatitis $\mathrm{C}$ patients with MELD score $>10$ have SSLR for HCC presence of 4.57 and are in need for closer follow-up.

\section{References}

1- SAAR B. and KELLNER F.: Radiological Diagnosis of Hepatocellular Carcinoma. Liver International, 28: 189 199,2008

2- ANWAR W.A., KHALED H.M., AMRA H.A., et al.: Changing pattern of hepatocellular carcinoma (HCC) and its risk factors in Egypt: Possibilities for prevention.

3- Egyptian national control strategy for viral hepatitis, 20082012 Arab Republic of Egypt, Ministry of Health and Population, National Committee for the Control of Viral Hepatitis.

4- MAZZAFERRO V., REGALIA E., DOCI R., et al.: Liver transplantation for the treatment of small hepatocellular carcinomas in patients with cirrhosis. N. Engl. J. Med., 334 (11): 693-699, 1996.

5- MALINCHOC M., KAMATH P.S., GORDON F.D., et al.: A model to predict poor survival in patients undergoing transjugular intrahepatic portosystemic shunts. Hepatology, 31: 864-871, 2000

6- KAMATH P.S., WIESNER R.H., MALINCHOC M., et al.: A model to predict survival in patients with end-stage liver disease. Hepatology, 33: 464-470, 2001.

7- WIESNER R.H.: Patient selection in an era of donor liver shortage: Current US policy. Nat. Clin. Pract Gastroenterol. Hepatol., 2: 24-30, 2005.

8- SHARMA P., HARPER A.M., HERNANDEZ J.L., HEFFRON T., MULLIGAN D.C., WIESNER R.H., et al.: Reduced priority MELD score for hepatocellular carcinoma does not adversely impact candidate survival awaiting liver transplantation. Am. J. Transplant, 6:1957-1962. [PubMed: 16771808], 2006.

9- WIESNER R.H., FREEMAN R.B. and MULLIGAN D.C. Liver transplantation for hepatocellular cancer: The impact of the MELD allocation policy. Gastroenterology, 127 (5 Suppl 1): S261-S267. [PubMed: 15508092, 2004.

10-WASHBURN K., EDWARDS E., HARPER A. and FREEMAN R.: Hepatocellular carcinoma patients are advantaged in the current liver transplant allocation system. Am. J. Transplant, 10:1643-1648. [PubMed: 20486906], 2010.

11- MASSIE A.B., CAFFO B., GENTRY S.E., HALL E.C., AXELROD D.A., LENTINE K.L., et al.: MELD Exceptions and Rates of Waiting List Outcomes. Am. J. Transplant, 11: 2362-2371. [PubMed: 21920019], 2011. 
12- SINGAL A.K., GUTURU P., HMOUD B., KUO Y.F., SALAMEH H. and WIESNER R.H.: Evolving frequency and outcomes of liver transplantation based on etiology of liver disease. Transplantation, 95: 755-760. [PubMed: 23370710], 2013.

13- OPTN \& SRTR: Annual Data Report. U.S. Department of Health and Human Services, Health Resources and Services Administration, Healthcare Systems Bureau, Division of Transplantation; Rockville, MD: Scientific Registry of Transplant Recipients, 2012.

14- New Recommendations Issued for Transplantation in Patients With Hepatocellular Carcinoma. Medscape. Mar. 17, 2010.

15- EASL-EORTC Clinical Practice Guidelines: Management of hepatocellular carcinoma. European Association for the Study of the Liver*, European Organisation for Research and Treatment of Cancer European Journal of Cancer, 48: 599-641, 2012.

16- POON R.T. and FAN S.T.: Evaluation of the new AJCC/UICC staging system for hepatocellular carcinoma after hepatic resection in Chinese patients. Surg. Oncol. Clin. N. Am., 12: 35-50, viii, 2003.

17- KAMATH P.S., WIESNER R.H., MALINCHOC M., et al.: A model to predict survival in patients with end-stage liver disease. Hepatology, 33: 464-70, 2001.

18- BECK J.R.: Likelihood ratios. Another enhancement of sensitivity and specificity. Arch. Pathol. Lab. Med., 110: 685-686, 1986.

19- SESHASAI S.R.K., KAPTOGE S., THOMPSON A., et al.: Diabetes mellitus, fasting glucose, and risk of causespecific death. N. Engl. J. Med., 364: 829-841, 2011.

20- YANG W-S., VA P., BRAY F., et al.: The role of preexisting diabetes mellitus on hepatocellular carcinoma occurrence and prognosis: A meta-analysis of prospective cohort studies. PloS One, 6: e27326, 2011.

21- ALCORN J.B.: United Network for Organ Sharing. Changes to OPTN by laws and policies from actions at November board of directors meeting. http://optn.transplant.hrsa. gov/media/1140/policy notice 12-2014.pdf. Published December 12, 2014.

22- AYMAN YOSRY, RABAB FOUAD, HANAN ABDEL HAFEZ, et al.: Transient Elastography can Predict the Risk of Hepatocellular Carcinoma in Egyptian Patients with Chronic Hepatitis. Journal of GHR July, 212 (7): 687-691, 2013.

\section{نموذج المرحلة النهائية لمرضى الكبد وسرطان الكبد}

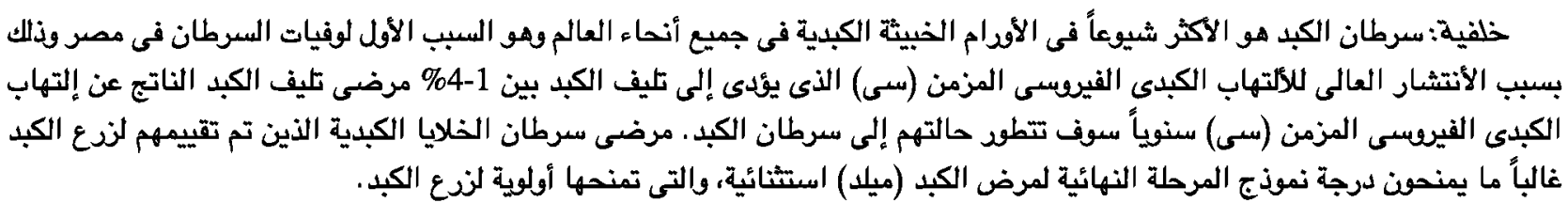

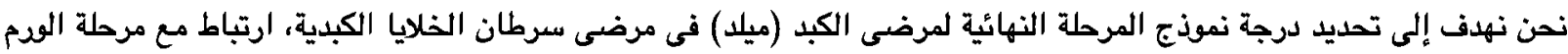

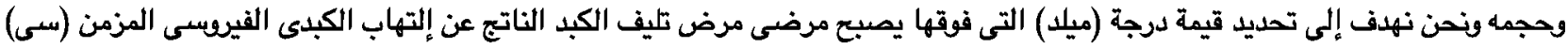
لايهم فرصة كبيرة لتطوير سرطان الخلايا الكبدية.

المواد والطرق: شملت الدراسات 98 من المرضى النين يعانن من الإلتهاب الكبدى الفيروسى (سى) مع سرطان الخالايا الكبدية (المجموعة

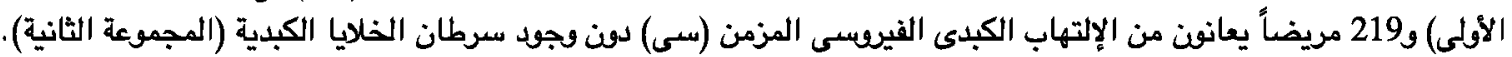

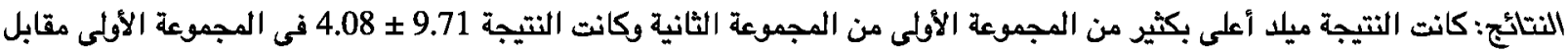

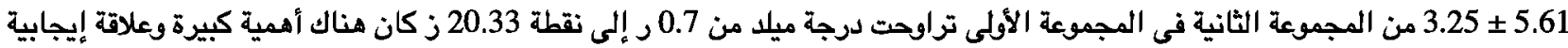

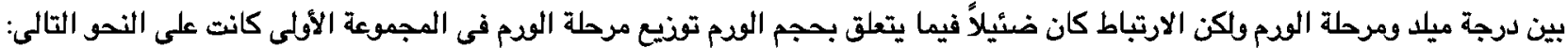

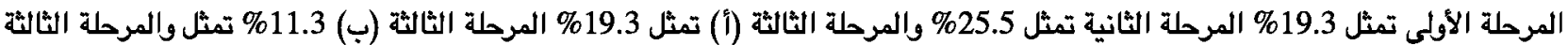
(ع) 1\% تمثل والمرحلة الرابعة (أ) 8\% تمثل والمرحلة الرابعة (ب) تمثل 7. 7.

الخلاصة: درجة نموذج المرحلة النهائية لمرضى الكبد (ميلد) لها علاقة إيجابية كبيرة مع مرحلة الودم فى حالات سرطان الكبد الكبد مرضيى

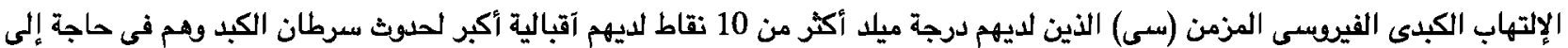
متابعة أوثق. 